


\title{
LA RESPONSABILIDAD CONTABLE EN LAS CRISIS ECONÓMICAS: CUESTIONES ÉTICAS
}

\author{
Autor: Francisco Luis Sastre Peláez. ${ }^{1}$ \\ Departamento de Gestión Financiera. \\ Universidad Pontificia Comillas.
}

\section{Resumen}

La calidad de la información contable reviste una extraordinaria importancia para el buen funcionamiento de las empresas y del sistema económico en su conjunto. Es por ello que su ejercicio está reservado a profesionales, es decir personas científicamente cualificadas, sometidas a un estricto código deontológico y orientadas en su actividad al bien público. El olvido o menosprecio del componente ético, imprescindible en el ejercicio de la profesión contable, así como de su naturaleza de servicio público da lugar a formas de contabilidad creativa muy nocivas para el buen funcionamiento de la economía.

En el presente artículo se analiza la naturaleza científica de la contabilidad, su utilidad pública y el indispensable componente ético que implica su ejercicio, así

\footnotetext{
${ }^{1}$ fsastre@cee.upcomillas.es
} 


\title{
Francisco Luis Sastre Peláez
}

como la responsabilidad que el mal uso de esta ciencia ha tenido en los escándalos financieros y en la gran crisis económica de principios del s.XXI.

Palabras clave: Ética contable, educación contable, ética de los negocios, escándalos contables, contabilidad creativa, crisis económica.

Accounting responsibility in economic crisis. Ethical issues.

\begin{abstract}
Accounting information has an extraordinary importance for the companies' good performance and the running of the economic system as a whole. This is the reason why its practice is restricted and allowed only to professionals. Accounting professionals are people technically qualified, but also obliged by a strict deontological code and devoted to public service. If this accounting profession's ethical component is forgotten, or its goal of public service undervalued, a kind of damaging "creative accounting" arises with very negative effects both for companies and for the entire economic system.

In this paper the scientific nature of accounting is analized, as well as its public utility and its key ethical issues. In addition, the responsibility for the bad use of this science in the financial scandals and the big economic crisis of the beginning of this century is reviewed.
\end{abstract}

Key Words: Accounting Ethics, Accounting Education, Business Ethics, Accounting Scandals, Creative Accounting, Economic Crisis.

\section{INTRODUCCIÓN.}

Las decisiones de directivos, inversores y stakeholders en general no se realizan directamente sobre la realidad sino, en gran parte, sobre la representación que de la realidad realiza la contabilidad. En su progresiva virtualización, la gestión económica se desarrolla cada vez más no en el mundo real, sino en la imagen del mundo que los gestores de la información diseñan. Si esta imagen fuera distorsionada, bien por malicia o bien por incompetencia, el funcionamiento del sistema económico. o al menos de una parte importante de él, se vería gravemente afectado. 
La verdadera importancia de este fenómeno no se percibe, en general, ni por la sociedad ni por los propios contables, lo que puede dar lugar a graves consecuencias económicas y sociales, como muestra la reciente oleada de escándalos financieros que desembocaron en la actual crisis económica. La reducción del riesgo de una repetición futura de sucesos similares exige, entre otras cosas, la correcta comprensión de la responsabilidad científica y ética que el ejercicio de la contabilidad conlleva.

La denominada "gran crisis contable" surgida a raíz de la doble quiebra EnronArthur Andersen en 2001 ha propiciado un profundo proceso de reforma especialmente dentro del ámbito científico de la contabilidad y, en menor medida, en lo referente a su ejercicio ético. Se ha comprendido bien la necesidad de perfeccionar las herramientas contables, pero quizá aun no lo suficiente la importancia de la conciencia y principios éticos en la mente de quien las maneja.

El objetivo de este artículo es mostrar como ética y contabilidad, que para la mentalidad actual constituyen dos campos de conocimiento distintos, no pueden actuar en la práctica separadamente. Una deficiente conciencia ética por parte de los gestores económicos, y muy especialmente de los contables, conduce a decisiones que, bajo la apariencia de profesionalidad, lesionan gravemente la confianza de los mercados y de la sociedad en general. Esta "crisis de confianza" es, precisamente, uno de los más graves síntomas que suele acompañar a las crisis económicas.

\section{CRISIS Y CONTABILIDAD.}

\subsection{La gran crisis financiera, económica y social de 2008.}

La denominada "Gran Recesión de 2008" (Rampel, 2010: 1), que se inició como una crisis financiera, se convertiría más tarde en económica cuando la congelación de los créditos afectó al sector productivo y en crisis social como resultado de las medidas de austeridad impulsadas por los gobiernos. Lo que naciera en EE.UU como un problema financiero y local, acabó globalizándose y dando lugar a la crisis mundial más grave desde la Gran Depresión de 1929.

No es posible hallar en el siglo XX un periodo de más de veinte años libre de crisis económicas, hecho que parece confirmar la conocida teoría de los ciclos o fluctuaciones periódicas de la actividad de Mitchell ${ }^{2}$. No hay acuerdo entre los distintos autores en cuanto a las causas de los ciclos, a su duración u otros detalles, pero todos coinciden en lo fundamental: en las economías de mercado la actividad se desarrolla en una sucesión alternada de periodos de expansión y

\footnotetext{
${ }^{2}$ Véase Mitchell, 1923.
} 
contracción. Aunque, bien sea por motivos estructurales, por la dinámica propia del sistema o por otras causas, las fases de depresión y crisis sean inevitables, sus efectos negativos pueden y deben controlarse. Comprender el proceso y desarrollar técnicas efectivas para gestionarlo serían tareas fundamentales en la política económica de cada país.

Conocido todo lo anterior resulta insólito que, a pesar de la magnitud de la crisis que se avecinaba nadie, ni institución ni profesional alguno, supiera o pudiera predecirla. La inmensa mayoría de los analistas económicos presentaban proyecciones optimistas incluso a comienzos de 2008 (Soim, 2012: 421). $\mathrm{Si}$, como decía Friedman, lo esencial de la ciencia es su capacidad para predecir acontecimientos (Friedman, 1953: 7), entonces es evidente que la presente crisis muestra el fracaso de la ciencia económica actual. Un fracaso que es, a la vez, de conocimiento y de gestión.

Cuando R. Coase habla de la ciencia económica de nuestros días, lo hace para lamentar su formalismo "elegante pero estéril", la distancia entre sus concepciones teóricas y la realidad. La ciencia económica es una "bolsa de herramientas", concluye, un conjunto de técnicas exitosas y útiles, pero inconexas. Los economistas se complacen demasiado en el uso de esas técnicas y es por ello que la ciencia económica apenas ha variado ni en sus principios ni en sus problemas desde Adam Smith. Se ha avanzado en los detalles mientras se permanece congelado en los paradigmas. Como consecuencia, podría explicarse hoy lo esencial de la economía usando como manual de texto alguna obra clásica escrita hace cien o doscientos años, algo impensable en cualquier otra ciencia (Coase, 2002: 4).

En resumen, el problema fundamental según Coase sería que:

“...los economistas no estudian el funcionamiento del sistema económico... un sistema con todas sus interrelaciones... El gran problema es su incapacidad para considerar al sistema como objeto de estudio." (Coase, 2002: 5)

En la gestación y desarrollo de la crisis los economistas han fallado en el conocimiento teórico de los fenómenos, en los modelos, pero también en la puesta en práctica de algunas de aquellas técnicas que constituyen, en palabras de Coase, el orgullo de la economía.

"La crisis fue, a la vez, por un fallo del mercado y por un fallo del gobierno... Los directivos tenían demasiada confianza en sus estrategias de asunción de riesgos. Los reguladores tenían también demasiada confianza en las medidas que habían tomado... En resumen, había una enorme distancia entre la verdad y lo que la gente creía que era verdad.” (Kling, 2010: 21-22)

Así pues, precediendo y acompañando en su evolución a la crisis económica, se detectan graves errores de conocimiento, pero también de actuación. En re- 
lación con esta última queda en entredicho tanto la aplicación del principio de racionalidad por los agentes económicos (Soim, 2012: 422), como la sujeción de sus actuaciones a principios éticos (Duska y otros, 2011: 9 y ss.).

Entrando más en el detalle, y dejando a un lado los aspectos generales a los que nos referíamos más arriba, esta doble crisis ética y de conocimiento que ha puesto de manifiesto la recesión, se refleja en dos importantes grupos de cuestiones:

a) Un problema de fiabilidad de la información económica, principalmente contable: los datos que manejaban tanto las empresas como las instituciones en los años anteriores a la crisis no reflejaban fielmente la situación de riesgo creciente en que numerosas entidades, principalmente bancarias y de seguros, estaban sumidas. Ello era debido, en parte, a defectos internos de la propia ciencia contable (Kothari y Lester, 2012: 340) y también, frecuentemente, a la alteración fraudulenta de las cuentas por parte de las propias empresas (Byrne, 2002: 45), (O'Reilly, 2011: 26).

Como resultado de ello se produjo un proceso de toma de decisiones equivocado tanto por parte de los inversores como por las instituciones encargadas de la supervisión de los procesos. Más adelante, cuando en rápida sucesión se fue poniendo en evidencia la larga serie de incompetencias, fraudes e irregularidades, la desconfianza se adueñó de los mercados, agravándose la crisis aun más.

b) Un problema de supervisión: tanto de la veracidad de la información contable elaborada por las empresas (revisión a cargo de las compañías de auditoría) como de la correcta actuación de los distintos agentes económicos y financieros (organismos públicos de supervisión y control) (Peláez, 2011:117), (Lowenstein, 2008).

Durante la crisis, y en los años inmediatamente anteriores, se produjo una larga serie de escándalos financieros que, en la mayoría de los casos, se apoyaron en la alteración fraudulenta de las cuentas y en la colaboración, dolosa o inadvertida, de las compañías auditoras e instituciones de supervisión.

La crisis de la contabilidad no esperó, para eclosionar, a la Gran Recesión del 2008. Lo hizo con la doble quiebra Enron-Arthur Andersen a finales del año 2001 (Byrne, 2002: 44). Se inició entonces un frenético proceso de revisión tanto de la normativa contable como de los códigos éticos profesionales de las compañías consultoras y de auditoría. Se intentaba reparar el gravísimo descrédito en que había caído la profesión contable, así como la sangría de clientes que amenazaba a las grandes empresas de auditoría con el mismo destino sufrido por Arthur Andersen. La sangría se detuvo, pero no se alcanzó a evitar que las quiebras por fraudes contables, con efectos sociales devastadores, continuaran creciendo en los años siguientes (Duska y otros, 2011: 14 y ss). 
En resumen: la gran crisis del 2008 no solo ha sido financiera, económica y social, sino también una crisis de confianza producida por la inadecuada gestión del conocimiento económico disponible y un bajo nivel de conciencia ética en las actuaciones. Como consecuencia de ello la actividad económica se contrajo, desplomándose la fe en ciertas instituciones y profesionales que ya venían siendo cuestionados a causa de numerosos escándalos financieros en los años anteriores. Las críticas se centraron, muy especialmente, en los individuos e instituciones que elaboran, supervisan, manejan o difunden información relevante sobre las empresas y el mercado: organismos de supervisión, agencias de rating, auditores y contables.

\subsection{La responsabilidad contable en las crisis económicas.}

Como ya hemos comentado antes, la desconfianza en la información divulgada por contables y auditores no surge con esta crisis económica sino que la antecede y acompaña constituyendo, además, uno de sus elementos agravantes.

En EE.UU., país donde se han dado los escándalos contables más significativos, las quejas por fraudes en las cuentas se habían venido produciendo a lo largo de todo el siglo XX (Benston, 2003: 3). A pesar de la creación de diversos órganos supervisores la sucesión de quiebras de empresas, escándalos y fraudes generados o favorecidos por una contabilidad engañosa o fraudulenta, lejos de detenerse, creció exponencialmente en las últimas décadas del siglo. Pero es en los primeros años de este siglo XXI cuando los fraudes contables, frecuentemente seguidos por despidos masivos y cierre de empresas, alcanzan el mayor nivel de frecuencia y gravedad.

Beneficios "inflados" de forma artificial, pérdidas encubiertas, malas prácticas de contabilización de activos y pasivos, etc. son las técnicas más frecuentes en los grandes escándalos de principios de siglo: Waste Management (1998), Enron (2001), WorldCom (2002), Tyco (2002), HealthSouth (2003), Freddie Mac (2003), American Insurance Group (2005), Lehman Brothers (2008), etc. Y, en lo que se refiere a España: Gescartera (2001), Afinsa y Forum Filatélico (2006), Martinsa-Fadesa (2008), etc.

En noviembre de 2001 se produce la quiebra de Enron, una de las mayores compañías eléctricas del mundo. Su hundimiento marcará un antes y un después en la "crisis de la contabilidad", crisis que anticipa la económica del 2008, y de la que se convertiría en uno de sus catalizadores.

La crisis de Enron fue importante por diversos motivos. En primer lugar por la magnitud económica de las pérdidas ocasionadas ${ }^{3}$, pero también porque supuso la quiebra de Arthur Andersen, por aquel entonces la principal compañía auditora del mundo. Arthur Andersen, auditor externo y consultor de Enron fue cómplice,

${ }^{3}$ Las pérdidas para los accionistas se estimaron en unos 82.000 millones de dólares (50.000 en patrimonio y el resto en capitalización bursátil) y para los empleados, aparte naturalmente de la pérdida de empleos, en más de 1.000 millones de dólares en fondos de pensiones (Byrnes 2002,44). 
o al menos encubridor, del entramado contable creado por esta compañía para ocultar durante años sus pérdidas millonarias.

El papel central ocupado por la falsa contabilidad de Enron en el fraude, así como la colaboración dolosa precisamente de la compañía encargada de certificar la validez de aquellas cuentas fue la gota que colmó un vaso ya por entonces demasiado lleno. La auditoria, una de las principales ramas de la contabilidad, cayó en un gran descrédito, al igual que la contabilidad misma, por la facilidad con que se podía proceder al "maquillaje" de sus cuentas. Es a esa facilidad a la que alude el término "contabilidad creativa" expresión que, con un significado peyorativo, pasa a ser de uso corriente a partir de aquellos años (Amat, 2004: 13).

Como ya dijimos, la sucesión de engaños contables, que ocasionaban gravísimas pérdidas a los inversores y empleados de las compañías, había sido continuada y, en los últimos tiempos, peligrosamente creciente. Se estima que en los seis años anteriores a la crisis de Enron, y solo en los Estados Unidos, se descubrieron y obligaron a corregir falsas contabilidades que ocasionaron a los accionistas pérdidas superiores a los 200.000 millones de dólares (Byrne, 2002: 44).

Tras la quiebra Enron-Arthur Andersen, y en un desesperado esfuerzo por recuperar la credibilidad del mercado, las compañías auditoras realizaron un proceso de profunda reforma en sus estructuras y formas de operar. A la vista de los importantes y reiterados escándalos contables se realizaron también acciones institucionales de reforma normativa que impidieran, en lo posible, su repetición futura, (Cernuska, 2011: 37) acciones que algunos criticaron por insuficientes (Duska y otros, 2011: 29).

Lo que resulta sorprendente, nos recuerda McPhail, es el rápido olvido social en el que fueron cayendo todos estos desastres. Y más sorprendente aún, recalca este autor, fue la falta de atención que se concedió al papel que en ellos representara el componente ético. En especial, la función que la ética profesional contable debería haber jugado en los procesos de falseamiento de cuentas fue una cuestión que pasó casi totalmente inadvertida, tanto al considerar el problema como cuando se arbitraron soluciones para prevenir su repetición. La razón de este hecho, razona McPhail, es probablemente que la práctica general de los negocios y, en concreto, de la profesión contable tiende a verse como una actividad amoral, dirigida ante todo por el principio de eficiencia (McPhail, 2009: 3 y ss.).

Como es sabido, la contabilidad es una disciplina integrada dentro del ámbito de la ciencia económica y que responde tanto en su estructura como en su funcionamiento a los fines y principios generales de aquella. Por otra parte, y como veremos después con más detalle, desde hace al menos un siglo la economía, en cuanto ciencia, viene proclamando tajantemente su carácter objetivo y su independencia de toda norma o razonamiento moral. Y al hacerlo así no actúa de forma abusiva o arbitraria sino que, por el contrario, adapta estrictamente su objeto de estudio a lo que hoy se entiende por científico. 
El problema surge, en nuestra opinión, porque la mentalidad general tiende a extender esta independencia de la norma moral no solo a los contenidos científicos de la economía, sino también a la aplicación práctica que de ellos hacen los profesionales. Como consecuencia de esto, no solo los principios y las técnicas en el ámbito del conocimiento, sino también las decisiones económicas y contables que toman las personas en el mundo real, dejarían de estar sujetos a los principios éticos. Este falso razonamiento, que nos aboca a considerar el principio del máximo beneficio económico como ley suprema de la acción humana, sería una de las principales razones por las que los principios éticos tienen tan escasa influencia en el ejercicio profesional de la Contabilidad ${ }^{4}$.

\section{ETICA Y CONTABILIDAD. ¿ES LA CUESTIÓNÉTICA UN PROBLEMA ECONÓMICO?}

\subsection{Fragmentación del conocimiento y evolución de las ciencias: economía científica y ética filosófica.}

Quizá la mejor y más hermosa definición del propósito de la economía es la que deduce Nielsen de la lectura de Aristóteles. Si, como se afirma en la Ética a Nicómaco, la actividad económica es una praxis que tiene por objeto la creación de riqueza, podríamos concluir que el objeto de la economía es, según Aristóteles:

"la creación de riqueza de forma que el que la realiza se convierta en mejor persona y el mundo en un mejor lugar para vivir" (Nielsen, 2010: 299).

En el proceso de transformación de la sabiduría económica en conocimiento científico desarrollado a lo largo del s. XIX, en algún momento entre Ricardo y Walras, se rompe la fusión aristotélica inicial entre objetivo económico (riqueza) y acción ética (perfeccionamiento humano). La economía se desgaja, definitivamente, de la filosofía. Con ello, el fin económico y la "praxis" ética quedan subordinados a disciplinas distintas. El fin económico, la creación de "riqueza", se vuelve mensurable al transformarse "riqueza" en utilidad, y utilidad en el valor en dinero que ofrece el mercado por las cosas, con lo que puede ser integrado sin dificultad en el ámbito de la ciencia. Por su parte la acción ética, el desarrollarse como persona y mejorar el mundo, intraducible en términos de medida, queda

\footnotetext{
${ }^{4}$ Diversas investigaciones demuestran que el nivel de razonamiento ético de los contables es inferior al de otros profesionales. Esto es así tanto en los contables con experiencia como en los estudiantes de contabilidad. Otros estudios parecen demostrar que el nivel de razonamiento ético de los estudiantes de esta disciplina disminuye a medida que avanzan en sus estudios. (McPhail, 2009: 4 y ss).
} 
abandonada en el seno de la filosofía, junto al resto de los conocimientos que no fue posible convertir en científicos (Dogan y Pahre, 1989: 61).

Si a finales del siglo XIX el divorcio entre economía y ética es casi completo, a mediados de la centuria siguiente cualquier conexión que hubiera podido existir entre estas dos disciplinas está ya completamente disuelta (Vickers. 1997: 5 y ss).

Friedman, en el mismo decisivo artículo en el que proponía que la validez de las teorías económicas radica en su capacidad predictiva, afirmará:

"la economía positiva es, en principio, independiente de cualquier posición ética particular" (Friedman. 1953: 4)

A pesar de las inquietudes sociales perceptibles en la obra de la mayoría de los grandes economistas, desde el propio Adam Smith hasta Gary Becker o Amartya Sen, en esta frase de Friedman se resume cumplidamente la posición ortodoxa mantenida por la economía en los últimos dos siglos. Lo que no impide, sin embargo, que en fechas recientes diversos autores aboguen por una reintegración entre economía y ética o, al menos, por un incremento de colaboración entre ambas disciplinas. Así, por ejemplo, V.Walsh afirma que uno de los más importantes avances en economía de los últimos tiempos ha sido: "el derrumbe de las barreras para un intercambio fructífero entre la teoría económica y la filosofía moral" (Vickers, 1989: vii).

¿Es conveniente la colaboración entre ética y economía? o, aun más, ¿sería recomendable la refundación de la economía para dar lugar a una nueva "economía ética”?

El proceso de fragmentación de las disciplinas es un fenómeno general, que no solo ha afectado a ética y economía, sino a todas las disciplinas tanto científicas como sociales. (Dogan y Pahre, 1989: 56). En la época en que Aristóteles creaba el concepto de economía al que nos hemos referido antes, la mayor parte del conocimiento humano formal se concentraba en una única disciplina, la filosofía, aunque en el seno de ésta ya comenzaran a latir algunas ciencias embrionarias. La fragmentación de la filosofía en disciplinas científicas independientes comenzaría con la aparición de las universidades, en la Alta Edad Media, continuando con la Revolución Científica del siglo XVII, para acelerarse después durante los siglos XIX y XX.

El fenómeno más destacado en la evolución histórica tanto de las disciplinas científicas como sociales, es el intenso proceso de fragmentación de las mismas y, a la vez, los numerosos intentos de recombinación a la hora de investigar campos híbridos (Dogan y Pahre, 1989: 57).

La razón del proceso de fragmentación de las disciplinas deriva directamente de nuestra forma de conocer y, en concreto, de la manera en que construimos la metodología científica. Para analizar un fenómeno en profundidad es preciso crear un sistema aislado. No obstante, los científicos sociales no pueden aislar los fenómenos en el laboratorio, por lo que se ven obligados a utilizar un método 
analítico. Cada disciplina social ha seleccionado ciertos fenómenos como objeto de su estudio y avanza explorando dichos objetos exclusivamente, y tan profundamente cómo es posible.

"Cada disciplina arroja luz sobre un conjunto de variables precisamente porque otros factores se asumen como externos, distales e imparciales" (Sartori, citado por Dogan y Pahre, 1989: 57).

Así pues, fueron desgajándose progresivamente de la filosofía todas aquellas áreas de estudio cuyos problemas se pudieron aislar, identificar y definir como susceptibles de conocimiento científico, y se abandonaron en su seno aquellas sobre las cuales no fue posible el acuerdo o la verificación (Piaget, 1970: 13). Una de estas últimas, tal vez la más importante, es la ética o filosofía moral.

En resumen, la separación entre economía y filosofía se produjo como condición necesaria para la aplicación del método científico a los problemas económicos y el consiguiente nacimiento de la ciencia económica.

No obstante, aunque la simplificación analítica, facilitada por la fragmentación y la especialización, es necesaria para clarificar y explorar con profundidad ciertas relaciones causales, su uso conlleva también importantes inconvenientes. La especialización es, por definición, autolimitante por lo que la información que los conocimientos especializados proporcionan de los fenómenos es necesariamente parcial y la colaboración entre disciplinas distintas (interdisciplinariedad) se vuelve imprescindible. Además, el avance de las ciencias pone cada vez más en evidencia la defectuosa delimitación entre ellas, lo que conduce a la hibridación ${ }^{5}$. (Dogan y Pahre, 1989: 57)

\section{2. ¿Ética del contable o ética de la contabilidad?}

El proceso de simplificación analítica, que hemos estudiado someramente en el apartado anterior, produce un distanciamiento entre el objetivo científico y la realidad. El conocimiento económico se incrementa, pero también se vuelve teórico, instrumental. Esta es precisamente, como recordábamos al principio de este estudio, la principal crítica que se hace de la ciencia económica actual (Coase, 2002: 5)

El objetivo de la economía es crear riqueza pero: ¿qué se entiende por tal?:y para quien o para qué crearla? La ciencia no explica la naturaleza de las cosas, o el fin de su existencia, sino que procede estrictamente a su delimitación, cuantificación y medida, determinando luego las posibles relaciones entre objetos distintos, o bien entre sus elementos constituyentes. El conocimiento científico es objetivo y neutro únicamente mientras permanece confinado en la ciencia que le dio origen, pero se convierte en medicina o veneno, arma o herramienta cuando, para aplicarse en el mundo, se transforma en tecnología.

${ }^{5}$ El término "hibridación" se refiere a la colaboración entre dos disciplinas distintas en el estudio de un subcampo muy específico situado en el límite entre ambas y difícilmente asignable en exclusiva a una $\mathrm{u}$ otra de ellas. 
En definitiva, la ciencia responde al cómo, pero no al quien o al para qué. Resolver esta última pregunta exige salir de la ciencia misma y entrar en el terreno de la filosofía.

$\mathrm{Si}$, como hemos visto, el desarrollo de la ciencia moderna implicó su separación de la filosofía moral, no es menos cierto que a la hora de actuar en el mundo físico no es posible aplicar el conocimiento científico sin tomar decisiones de orden moral.

Si la ciencia económica moderna, por definición, no es sujeto ético, la acción económica siempre lo es, al igual que el economista que la realiza y que el sistema, organismo o institución en cuyo nombre la acción se lleva a cabo.

\section{LOS COMPONENTES ÉTICOS DE LA PROFESIÓN CONTABLE.}

Partiendo del carácter neutro de la economía en cuanto ciencia, lo que cabe analizar desde un punto de vista ético es la actividad económica y, en concreto, la actividad contable.

A la hora de iniciar nuestro estudio hemos de considerar en primer lugar que, por su trascendencia y complejidad, en la mayor parte de los países el ejercicio de la contabilidad no es libre, sino que está reservado a profesionales.

El carácter de "profesional" implica, por si mismo, ciertas obligaciones morales que deberemos analizar. Del mismo modo, el ejercicio de la contabilidad acarreará otras obligaciones, que cualificarán a las primeras. Nuestro estudio ha de referirse, por consiguiente, a los contenidos éticos que conlleva el ejercicio de la profesión contable.

Según Duska, la ética es la disciplina que se ocupa de identificar lo que es correcto o incorrecto, lo que es bueno o malo. Su tarea es el análisis y evaluación de las acciones humanas. Aunque más tarde este autor precisa que por "acciones humanas" no solo hemos de entender actos aislados, sino también a las organizaciones, instituciones e incluso sistemas sociales, políticos o económicos en los que las acciones se realizan y que, de alguna manera, las canalizan y condicionan. El hecho de que tras los recientes escándalos económicos de todas partes se reclamara una reforma de la profesión contable implica, en sí mismo, un juicio ético. (Duska y otros, 2011: 34 y ss).

Si las acciones de la contabilidad no fueron buenas, eso significa que la profesión contable no cumplió con sus obligaciones morales, haciéndose con ello culpable, al menos en su condición de colaborador necesario, de los fraudes y crisis que se desencadenaron después.

Pero ¿cuáles son exactamente esas obligaciones éticas incumplidas por los contables? Seguiremos, en este punto, el análisis realizado por McPhail. (McPhail, 2009: 132 y ss) 
Como decíamos al principio de este epígrafe, aunque la legislación es distinta según los países, en la inmensa mayoría de ellos el ejercicio de la contabilidad no es libre. Está controlado por ciertas instituciones y reservado a personas que cumplan determinados requisitos. Es por ello que la mayoría de los contables, y de las compañías de auditoría, se presentan a sí mismos como "profesionales".

Los términos "profesión" y "profesional" tienen un significado profundamente social, pues nacen en referencia a la satisfacción de alguna necesidad de la comunidad. Los profesionales son personas con un mismo conocimiento de base, una misma educación y comprometidos con un mismo servicio público.

Por tanto, una "profesión" es una comunidad moral:

a) que actúa en interés de la comunidad (interés público),

b) cuyos miembros se distinguen como individuos y como grupo (independencia)

c) por un conjunto compartido de fines y creencias sobre los valores de dichos fines (código de conducta profesional).

Evidentemente, las obligaciones éticas específicas de cada profesión, en este caso de la profesión contable, vendrán cualificadas por la función para la que han nacido. Pero, en todo caso, el ejercicio de dicha función deberá realizarse de manera profesional, es decir, en interés público, con independencia y observando los códigos de conducta del grupo profesional.

¿Cuál es la función pública de la contabilidad? La contabilidad es una disciplina que se desarrolla eminentemente en el ámbito empresarial y que colabora a desarrollar las funciones y objetivos de la empresa. Debemos entonces analizar cuáles son estas funciones y qué papel cumple la Contabilidad en su realización.

\section{FUNCION DE LA EMPRESA Y NECESIDAD DE LA CONTABILIDAD.}

\subsection{Función de la empresa.}

La empresa surge como una colaboración entre personas y grupos económicos de interés (stakeholders económicos ${ }^{6}$,en adelante stakeholders) y existe mientras esa relación de colaboración se mantiene. La función de la empresa es satisfacer a to-

\footnotetext{
${ }^{6}$ Entenderemos el término "stakeholder" desde una perspectiva económica, es decir con un sentido restringido. A efectos de este articulo stakeholder económico, en adelante stakeholder, es toda persona interesada económicamente en la empresa. Es decir, vinculada a la empresa por una o más transacciones económicas. El término podría llegar a abarcar, por adición, a todos los individuos de una sociedad, pero discriminados según el grupo de interés al que cada individuo pertenezca. Distinguiremos, principalmente: accionistas, directivos, empleados, clientes, proveedores y organismos públicos.
} 
dos sus stakeholders, y no solo a algún grupo privilegiado de ellos. Por ello afirmar, por ejemplo, que el objetivo empresarial es la producción de bienes útiles para la sociedad (clientes), o crear beneficio para sus socios (accionistas) es ofrecer una explicación incompleta. La función de la empresa es crear beneficio (valor o utilidad) para todos sus stakeholders.

El stakeholder aporta recursos a la organización buscando compensaciones futuras que le supongan un beneficio privado. Para ello acepta ciertas "reglas del juego", que consisten esencialmente en lo siguiente: la totalidad de los recursos aportados se gestiona por un cierto grupo de interés (directivos), combinándolos en un proceso de creación de valor (explotación) con el fin de obtener un beneficio común, que se repartirá oportunamente entre todos los que han participado en el proceso.

Nótese que el procedimiento consiste en obtener primero un beneficio común, del que se detraerán y repartirán luego los beneficios particulares para los que participan en él. Su buen funcionamiento implica que se cumplan dos condiciones:

1.- que el individuo trabaje en primer lugar para el grupo (la empresa), y no para sí mismo, aunque en su pensamiento esté el objetivo final de conseguir con ello un beneficio personal.

2.- que la empresa, a través de las personas encargadas de su gestión, compense a cada stakeholder por sus aportaciones y reparta equitativamente el beneficio obtenido.

\subsection{Necesidad de la contabilidad.}

La obtención del resultado común es una tarea azarosa y que conlleva tiempo, por lo que hasta que se produce y se procede a su reparto, cada stakeholder precisa información sobre la forma en que se va gestionando su aportación. Especialmente estará interesado en el beneficio y patrimonio que se va obteniendo con los recursos comunes, con el fin de obtener respuesta a dos preguntas: cuál será su ganancia y si la empresa tendrá capacidad material (liquidez) para abonársela en el momento acordado. Si la información necesaria no se le ofrece o si ésta no fuera satisfactoria, el stakeholder interrumpirá su colaboración y la empresa habrá de sustituirlo o, si no lo lograra, cesar en su actividad. Suministrar la información necesaria es, entonces, una tarea imprescindible para la continuidad empresarial.

La enorme masa de acontecimientos que vive la compañía día a día, así como la dificultad de su identificación, valoración y posterior presentación de forma inteligible, exige la realización de múltiples tareas especializadas y complejas con el fin de elaborar la información requerida. Como es sabido, la ciencia que se ocupa de realizar esas tareas es la Contabilidad y los documentos en los que queda resu- 
mida la información contable para uso de los stakeholders se denominan "estados financieros". Anualmente se hace pública una selección de los principales estados financieros (Cuentas Anuales) para uso de los stakeholders, actuales o futuros, no vinculados directamente a la gestión de la empresa.

Como la complejidad de la tarea encomendada a los contables es grande, el riesgo de que la información ofrecida por éstos no describa adecuadamente la realidad de la empresa es elevado. Es por ello conveniente subdividir a las personas relacionadas con la contabilidad en dos grupos:

a) los contables: son los responsables de crear la información necesaria a partir del registro y valoración de los fenómenos de interés económico que afecten a la empresa.

b) los auditores: su función será supervisar la información ofrecida por los contables, verificando que en su creación se han respetado los procedimientos establecidos, y que no existen en ella errores o debilidades de control.

Los contables suelen formar parte del personal en plantilla de las empresas para las que trabajan. En cuanto a los auditores, lo normal es que se integren en compañías de servicios especializadas, cuya función social es revisar las cuentas de las empresas clientes.

\section{FUNCIÓN DE LA CONTABILIDAD.}

\subsection{Introducción.}

Como ya dijimos, la función de la contabilidad es suministrar a los stakeholders de la empresa, la información que necesitan para tomar sus decisiones, información que se dirige a contestar dos preguntas:

1.- ¿Cuál será el resultado empresarial?

2.- ¿Dispondrá de fondos la empresa para pagar mi participación en el proceso?

La segunda pregunta afecta a todos los stakeholders, sea cual sea la naturaleza de su prestación y forma de retribución. La primera, sin embargo, interesará sobre todo a aquellos en los que la cuantía de su retribución dependa en todo o en parte del resultado obtenido. La información necesaria para responder a estas dos preguntas la proporciona la denominada contabilidad financiera, una de las dos ramas principales en las que se subdivide la contabilidad empresarial.

Por otra parte, los stakeholders a los que se haya asignado responsabilidad en la obtención del resultado (directivos y gestores en general) necesitarán infor- 
mación adicional que les permita la gestión y optimización de aquél. Esta información, centrada en el análisis del resultado de las operaciones normales de la compañía (explotación), la proporciona la contabilidad analítica o de gestión, la segunda de las dos grandes ramas de la contabilidad.

\subsection{El principio fundamental de la Contabilidad: la "imagen fiel".}

En España la principal norma reguladora de la contabilidad financiera es el Plan General de Contabilidad del año 2007 (PGC 2007)7, en el que se establece que el objetivo principal de la Contabilidad es ofrecer una "imagen fiel de la empresa”. La expresión "imagen fiel”, de larga tradición sobre todo en Reino Unido y sus áreas de influencia, hace referencia a una visión de la compañía que sea objetiva, sin engaños, para que permita a los interesados hacerse una idea cabal del desempeño de la organización y tomar decisiones sobre ella no sesgadas por una información deficiente. Así, en su primera parte, epígrafe 1, el PGC 2007 establece:

"Las cuentas anuales deben redactarse con claridad, de forma que la información suministrada sea comprensible y útil para los usuarios al tomar sus decisiones económicas, debiendo mostrar la imagen fiel del patrimonio, de la situación financiera y de los resultados de la empresa..."

Y un párrafo después se afirma que dicha imagen fiel se conseguirá mediante la aplicación sistemática de los principios y criterios contables desarrollados en el propio Plan, con lo que se coloca al principio de imagen fiel como objetivo principal y referencia última de la norma.

La sencillez y claridad de este principio es solo aparente. El origen del término es antiguo e impreciso y, a pesar de su uso continuado, nunca fue definido (Socias, 2008: 45). Más que de un principio se trata de un objetivo, de una meta a alcanzar. Y el propio PGC 2007 parece sostener lo mismo cuando afirma que la forma de lograr la imagen fiel en las cuentas anuales es la aplicación puntual del resto de principios y criterios contenidos en el Plan.

Por otra parte, no es posible regular únicamente mediante normas la diversidad y complejidad de situaciones que se presentan a la contabilidad en el día a día de las empresas. Es por ello que existen en todos los países una variedad de organizaciones profesionales que, por un lado, interpretan y completan dichas normas y, por otro, establecen un modelo ético de comportamiento a sus miembros por medio de códigos deontológicos.

\footnotetext{
${ }^{7}$ Ministerio de Economía y Hacienda (2007) Plan General de Contabilidad. Real Decreto 1514/2007, de 16 de noviembre. BOE, Suplemento del número 278. Año CCCXLVII, 20 de noviembre de 2007.
} 
Así pues, y resumiendo, la función básica de la contabilidad consiste en ofrecer, a través de sus documentos, una imagen fiel de la empresa, de su patrimonio y resultados, con el fin de ayudar a sus usuarios (stakeholders) en la toma de decisiones económicas. Para ello, el contable ha de aplicar diligentemente las disposiciones normativas aplicables en su país, así como los principios y reglas recogidos en los códigos éticos de su profesión.

\section{OBLIGACIONES ÉTICAS DE LA ACTIVIDAD CONTABLE.}

Como resumen de lo tratado en los epígrafes anteriores e introducción de lo que veremos en este, podríamos decir con Duska:

"La profesión contable se desarrolló para dar una verdadera y ajustada representación de los asuntos financieros de las organizaciones. Esa representación es importante en diversos ámbitos. Su exactitud es crucial. La creación de representaciones inexactas realizadas para explotar a aquellos con legítimo derecho a conocer la verdadera representación es equivalente al comportamiento no ético de la mentira." (Duska y otros, 2011:30)

En la cita anterior Duska nos ayuda a retomar y precisar algunas cuestiones de importancia:

a) por un lado nos recuerda que la actividad contable es profesional, y que consiste en la producción de información veraz sobre la empresa. Esta "manifestación veraz" (true disclosure) sería la principal exigencia ética a la contabilidad, y su principal dificultad moral.

b) Además, el hecho de ser una actividad profesional implica asumir otras obligaciones: actuación en función del bien público, independencia y respeto a los códigos de conducta profesionales.

Por otro, que no todos tienen derecho a recibir dicha información. La información contable, que siempre deberá ser veraz, no necesariamente deberá emitirse en cualquier ocasión o para todo el mundo. La obligación del contable, si bien inspirada en el bien público, no es hacia toda la sociedad de forma uniforme e indiscriminada, sino hacia los stakeholders. La naturaleza de cada stakeholder, y especialmente su responsabilidad o no en el proceso de gestión, condicionará el tipo de información a la que ha de tener acceso y el momento en que deberá recibirla. Por ejemplo, cualquier stakeholder recibirá la información de la contabilidad financiera (cuentas anuales), pero solo los stakeholders gestores tendrán derecho a obtener también la información de la contabilidad de gestión. 
Veamos más en detalle las cuatro obligaciones principales de la actividad contable:

\section{1. "Manifestación veraz".}

La primera obligación de la contabilidad es cumplir éticamente con la función para la que fue creada, es decir, proporcionar a los stakeholders una imagen fiel de la empresa.

No obstante, a causa de la relatividad e imprecisión del concepto de "imagen fiel" y de la dificultad de medición precisa de ciertos hechos económicos, se origina dentro de la contabilidad un amplio campo para la divergencia de opiniones en lo que se refiere a la mejor forma de representar algunas operaciones. Del mismo modo que dos personas describirían de diferente manera un mismo hecho del que fueron testigos, dos contables pueden diferir también en su descripción económica de una misma operación empresarial. La normativa contable pretende asegurar, sin conseguirlo del todo, que las discrepancias en la elaboración de las cuentas por los diferentes profesionales sean lo menos relevantes posible.

Estas circunstancias han dado ocasión al nacimiento de la denominada "contabilidad creativa", una actividad a la que hemos aludido antes y que está adquiriendo una gran importancia en los últimos años (Amat, 2004: 10).

Naser $^{8}$ define la contabilidad creativa como un proceso de manipulación contable, aprovechando los vacios o deficiencias de la normativa, con el fin de transformar las cuentas "de lo que tienen que ser en lo que los que las preparan prefieren que sea".

La contabilidad creativa no es ilegal, ya que se aprovecha de los defectos de la normativa sin vulnerarla, pero si atenta contra los principios éticos al privilegiar indebidamente el interés privado de quienes la aplican a costa del interés público del total de los usuarios. Se trata, en realidad, de un fraude, aunque en la mayoría de los casos no sea punible? .

En relación con la obligación de "manifestación veraz", la responsabilidad del contable se refiere fundamentalmente a garantizar dos cuestiones:

a) la corrección técnica de las cuentas: Dada la complejidad de la contabilidad y los problemas que ha de resolver, la corrección nunca puede asegurarse del todo. La contabilidad creativa se produce, precisamente, porque suelen existir varias alternativas para representar cada hecho económico y opiniones diversas sobre la aplicabilidad especifica de los distintos principios y técnicas. Es esta "holgura" la que da origen a la imperfección de las

\footnotetext{
${ }^{8}$ Naser, citado por Amat (Amat, 2004:10)

${ }^{9}$ Las razones para la contabilidad creativa, así como una argumentación a favor y en contra de su uso puede leerse en Gowthorpe. (Gowthorpe, 1998: 28 y ss.)
} 
normas contables y a las posibilidades de presentar de varias formas distintas la situación económica o los resultados de una empresa.

La competencia que se exige al contable para integrarse en la profesión intenta maximizar la calidad técnica de su trabajo. Por otra parte, y como es sabido, una vez finalizada la creación de las cuentas, se pide a profesionales contables independientes (compañías auditoras) que las revisen y atestigüen su "razonabilidad". Mas allá no podrían llegar, pues las "holguras" a las que nos referíamos antes existen tanto para el contable como para el auditor.

b) su no manipulación: es decir, que no se ha aplicado en su elaboración técnicas de "contabilidad creativa" (vulnerando la ética), ni mucho menos una contabilidad fraudulenta (vulnerando a la vez ética y legalidad).

Como el objeto de la manipulación de las cuentas es la búsqueda de beneficio privado por parte de algunos stakeholders, generalmente los gestores o administradores de la compañía, en perjuicio de los intereses de los demás, es conveniente analizar esta cuestión en relación con la segunda obligación ética del contable, es decir, con la búsqueda del interés público.

\subsection{Búsqueda del interés público.}

La profesión contable proclama que trabaja por interés público, y así se recoge en los códigos profesionales de numerosos países. Es decir, afirma que lo que hace es bueno no solo para un grupo de personas o de compañías, sino para la sociedad en general. No obstante cabe preguntarse, ¿en qué sentido es bueno su trabajo para la sociedad?

Si bien todas las profesiones nacen, se desarrollan y conservan en el tiempo su sentido en relación con la satisfacción de alguna necesidad social, es evidente que no todos los individuos pueden exigir el mismo tipo de prestación. La información contable es de naturaleza económica, y se refiere en general a entidades privadas, por lo que solo tendrán derecho a ella los vinculados económicamente a la entidad de referencia (stakeholders).

No obstante, la propia naturaleza y actividad de las compañías hace que, frecuentemente, sus stakeholders constituyan la práctica totalidad de los individuos o empresas de una comunidad, pero no en tanto miembros de dicha comunidad, sino en su condición de clientes, proveedores, accionistas o inversores. La empresa mantiene, además, una relación económica con al Estado, al recibir de éste ciertos servicios de naturaleza pública (seguridad jurídica, vigilancia policial, infraestructuras, etc.) que ella retribuirá principalmente por vía tributaria.

La empresa tiene otras obligaciones frente a la sociedad en general, sus instituciones, organizaciones, etc. que derivan no de transacciones comerciales, sino de 
su condición de miembro de la comunidad en la que opera. Estas obligaciones, al carecer de naturaleza económica, no cualifican a "la sociedad", las instituciones sociales, etc. como stakeholders, ni les otorgan derecho a recibir información contable. Por otra parte, las relaciones jurídicas, sociales, etc. que dan origen a esas obligaciones no podrían, hoy por hoy, ser objeto de la Contabilidad, al ser éste un lenguaje especializado exclusivamente, en transacciones de contenido económico.

La contabilidad deberá actuar, entonces, en función del bien público, pero considerando a éste no de forma uniforme, general y abstracta, sino como el bien de todos los grupos con los que la empresa mantiene una relación económica. "Bien público" será entonces el bien de todos sus stakeholders, agrupados e identificados según el tipo de relación económica que mantienen con la empresa.

Con el fin de asegurar, en lo posible, la actuación eficiente y productiva de la contabilidad en favor de los stakeholders, la doctrina estima que debe garantizarse la competencia del contable, entendida ésta a la vez en su vertiente técnica y como condición moral y de carácter ${ }^{10}$.

El principal problema ético que se plantea en relación con esta obligación es, precisamente, la manipulación de la información con el fin de privilegiar a algunos individuos o grupos de interés a costa de otros. Esta discriminación entre stakeholders es la piedra angular sobre la que se sostienen la práctica totalidad de los fraudes contables.

Los ejemplos son innumerables: gestores que alteran las cuentas con el fin de engañar a los inversores o defraudar en el pago de impuestos al fisco, directivos o socios que pretenden lucrarse ilícitamente a costa de los clientes y/o el resto de los accionistas, empresarios que buscan financiación y ofrecen una imagen engañosa de la empresa a los posibles financiadores, etc.

La manipulación de las cuentas rara vez surge por iniciativa del contable mismo y, aunque sea éste quien materialmente realice la alteración, su responsabilidad es más bien la de colaborador necesario que la de autor.

Enfrentado al conflicto de intereses entre el gestor que planea el fraude, y que normalmente es uno de sus superiores directos en el organigrama, y el resto de los stakeholders, el contable ha de elegir entre servir a "quien le paga" o a aquellos a los que, en numerosos casos, ni conoce siquiera.

Aunque el principio ético de servir al interés público estuviera claro para el contable, que frecuentemente no lo está, un problema aún más difícil se plantea a la hora de su aplicación práctica. ¿Cómo se puede decir no a un jefe directo, representante de la empresa, a quien se debe lealtad y del que depende el propio trabajo? Esta cuestión nos conduce al tercer principio ético de la contabilidad: la independencia.

10 Los problemas que plantea el definir en que consiste dicha competencia técnica y, sobre todo, cuáles son los rasgos de carácter necesarios son analizados por McPhail. (McPhail, 2009: 137 y ss) 


\subsection{Actuación independiente.}

En opinión de McPhail, la obligación profesional de independencia es considerada por numerosos autores y códigos deontológicos profesionales como una de las más importantes en relación con la profesión contable. No obstante, a la hora de desarrollar lo que dicha obligación significa, las discusiones adoptan un punto de vista muy estrecho y se ocupan solo de cuestiones prácticas como, por ejemplo, la separación entre relaciones de negocios y profesionales, el no servicio simultáneo a clientes cuando pudiera haber colisión de intereses, separación de servicios de auditoría y de consultoría, etc. (McPhail, 2009:142 y ss).

El verdadero problema de fondo, del que el conflicto entre el contable y el gestor del epígrafe anterior pudiera ser un ejemplo, permanece sin una respuesta satisfactoria. El ejercicio de la contabilidad no es autónomo, pues tanto los que la crean como los que la supervisan son contratados por el mismo grupo profesional: los directivos de las empresas.

Apelar, para mantener la necesaria independencia contable, a "actuaciones heroicas" de los profesionales, fortalecidos en la confianza interior de estar haciendo el bien y sin parar en consecuencias (Duska y otros, 2011: 74) no parece razonable. El comportamiento ético ha de ser cotidiano e impregnar los actos corrientes. Lo "heroico" sin embargo, por su propia naturaleza es extraordinario, tanto en lo que se refiere a las personas (pocos pueden ser héroes), como a los actos mismos (pocos actos del "héroe" son estrictamente "heroicos"). Querer realizar lo cotidiano mediante lo extraordinario es engañarse, y acabar conformándose a que jamás se lleve a cabo.

La situación de dependencia económica de la profesión contable, junto a la escasa conciencia por parte de la sociedad de la importancia que su actividad tiene para el buen funcionamiento de la economía son, creemos, dos de los principales problemas en relación con la crisis de la contabilidad. Varias de las propuestas de mejora sugeridas a partir de la eclosión del caso Enron han ido en el sentido de incrementar su independencia, al menos en lo referente a las compañías auditoras. No obstante, las iniciativas han quedado en el nivel superficial al que hacía alusión Duska ${ }^{11}$. (Duska y otros, 2011: 29).

En cuanto a las asociaciones contables el problema es garantizar su autonomía (evitando interferencias del Estado, por ejemplo), asegurando al mismo tiempo la independencia individual de los miembros y la calidad de la información emitida.

Así, por ejemplo, en el Reino Unido, también a raíz del caso Enron, se produjeron multitud de presiones para que las organizaciones profesionales contables

${ }^{11}$ Duska, citando a S. Gellerman, establece las cuatro racionalizaciones más corrientes para justificar por parte de los gestores y contables un comportamiento poco ético: a) "mi comportamiento no es realmente ilegal o inmoral", b) "las acciones se hacen en busca del mayor interés para mi compañía", c) "Nadie se enterará jamás" y d) "La compañía me protegerá" (Duska, 2011: 140 y ss). 
cambiaran su naturaleza puramente asociacionista y orientada al mercado a otra de carácter corporativo en la que el control del Estado tuviera más peso específico sobre la actividad contable. Se dotaría asi de mayor poder político a las organizaciones profesionales privándolas de parte de su autonomía. Esta corriente de opinión fue, sin embargo, contestada por los partidarios de mantener en las asociaciones profesionales una independencia a ultranza (McPhail, 2009: 135)

\subsection{Atenerse a los códigos éticos de la profesión.}

Una de las características esenciales de una profesión es la existencia de un código ético por el que se autorregulan (Claypool,1990: 699). En lo que se refiere a la contabilidad, y especialmente en el ámbito anglosajón, han aparecido en las últimas décadas diversas instituciones de profesionales independientes que han desarrollado pormenorizados códigos éticos. Uno de los de mayor prestigio e influencia es el publicado por el International Ethics Standards Board of Accountants (IESBA, 2013).

Los códigos éticos contables están generalmente vinculados a un sistema de cumplimiento basado en la vigilancia mutua entre los miembros de la profesión, incentivándose el envío de quejas a los colegios profesionales cuando se detectan infracciones. La experiencia demuestra, sin embargo, que este sistema de control no funciona (McPhail, 2009:145).

Los códigos tienen una función dual, sirven a la vez intereses propios de la profesión e intereses públicos, creando un sentido social en el miembro, pero a la vez justificando la necesidad de la profesión para la comunidad.

Aunque los hay de distintas clases (aspiracionales, educacionales, regulatorios...), en la mayoría de los institutos profesionales contables los códigos tienen dos partes o componentes: una primera en la que se recogen los principios y políticas generales de actuación y otra conteniendo las "reglas de cumplimiento".

En principio cabría pensar que el respeto a lo establecido en los códigos de conducta garantizaría una actuación profesional ética. No obstante, esta afirmación es cada vez más cuestionable. En el caso de la contabilidad, se observa en los últimos tiempos un cambio significativo en el enfoque de los códigos éticos. En ellos se ha ido sustituyendo el énfasis en la responsabilidad moral derivada del concepto de bien público por las especificaciones técnicas referentes a los servicios.

Por lo demás, la evidencia empírica sugiere que los códigos éticos tienen un escaso impacto en los profesionales ( (McPhail, 2009:147). Es un hecho significativo que la compañía Enron tuviera un código ético de 65 páginas, cuya portada era una carta de su fundador, K. Lay, afirmando que la empresa realizaba sus negocios "de acuerdo con todas las leyes en vigor y de forma moral y honesta" (Cernusca, 2011:36). 


\section{RESUMEN Y CONCLUSIONES.}

La larga serie de escándalos financieros que precedió y acompañó a la "Gran Recesión del 2008", así como la gestión y desarrollo de la crisis misma, evidenciaron graves carencias en la ciencia económica, tanto en su aspecto teórico como práctico. La crisis no fue solo económica, financiera y social, sino también de conocimiento científico y de credibilidad profesional.

Una de las áreas en que más significativamente se manifestaron estas carencias fue la relacionada con la información contable y su fiabilidad, tanto en el proceso de elaboración por las empresas interesadas como en su auditoria posterior.

La doble quiebra Enron-Arthur Andersen en el año 2002 marca el punto culminante de la crisis de confianza en la actividad contable, crisis que cabe atribuir a dos causas principales: por un lado a los defectos y limitaciones de la contabilidad misma y, por otro, al escaso peso del componente ético en su aplicación práctica profesional. El presente trabajo se centra, sobre todo, en la segunda de estas causas.

En algún momento del siglo XIX, al constituirse como ciencia independiente, la economía se vio obligada a renunciar a sus conexiones tradicionales con la filosofía moral. Se produce desde entonces una clara diferenciación, a efectos de conocimiento e investigación, entre economía y ética. No obstante, el carácter objetivo de la ciencia económica se extiende progresivamente a su aplicación práctica, llegando a considerarse la toma de decisiones y, en general, los actos económicos como "amorales" o éticamente "neutrales".

Es evidente que cualquier actividad humana, también la contable, tiene efectos buenos o malos en la vida de las personas. Por ese motivo, y en la medida en que la actividad sea fruto de la voluntad de los individuos, puede y debe ser objeto de juicio ético.

La actividad contable se desarrolla por profesionales y dentro de un ámbito económico-empresarial. Su objeto es suministrar a los stakeholders la información que necesitan para la toma de decisiones, una información que ha de caracterizarse necesariamente por reflejar fielmente la realidad económica de la organización a la que se refiere. Es lo que se denomina "manifestación veraz"(true disclosure) o "respeto del principio de imagen fiel". Así pues, cualquier falseamiento interesado de las cuentas (por ejemplo, la denominada "Contabilidad Creativa") vulnerará el principio de "manifestación veraz", referencia ética principal de la contabilidad.

Derivados del carácter profesional de la actividad contable, y profundamente relacionados con el principio de manifestación veraz, encontramos otros tres más: "servir al bien público", "independencia de actuación" y "respeto a los códigos éticos profesionales".

La práctica totalidad de los comportamientos no éticos en el ámbito de la contabilidad se generan por anteponer el bien privado de quien los realiza al 
interés público de los stakeholders. El medio utilizado para ello es el falseamiento de las cuentas, con lo que se quebrantan simultáneamente los principios de "bien común” y de "manifestación veraz".

La vulneración del principio de independencia es frecuente cuando el contable no es el autor principal del fraude, sino solo un colaborador necesario. Es muy difícil respetar este principio cuando el profesional, bien en su actividad de empleado en plantilla o de auditor independiente, depende económicamente del defraudador.

Por último, los códigos deontológicos o códigos éticos de la profesión, intentan aportar principios y reglas adicionales de comportamiento a los profesionales contables. No obstante, el carácter predominantemente ético que caracterizaba a estos códigos en otros tiempos ha ido perdiéndose, y se van asemejando cada vez más a "manuales técnicos de producto o servicio".

Creemos que la mayoría de las causas contables que han colaborado a crear la larga serie de desastres empresariales a los que antes hemos hecho referencia, siguen estando activas. Las reformas realizadas se han dirigido sobre todo a mejorar los aspectos técnicos de la contabilidad (depuración y cambio de normativa, creación de nuevas disposiciones, etc.) o de la estructura y relaciones comerciales de las compañías (especialmente consultoras y de auditoría), pero rara vez a los componentes éticos implicados en su ejercicio profesional. En este último aspecto, y salvo un reverdecimiento de las discusiones e investigaciones en ética contable, las iniciativas han ido poco más allá de la modificación de los códigos éticos profesionales de algunas compañías. Unos códigos que, aparte de su deriva a cuestiones más técnicas que éticas, tienen escaso seguimiento y un poder dudoso de influencia sobre el comportamiento de los profesionales.

Algunas medidas que, en nuestra opinión, ayudarían a avanzar en la solución de los problemas pendientes relativos a la ética contable serían:

1. Dar más poder político y social a las instituciones y asociaciones profesionales contables, con el fin de que pudieran desarrollar, por un lado, una protección más efectiva de los intereses de sus miembros y, por otro, un mejor control de la conducta de éstos. El apoyo de estas asociaciones ayudaría al contable a preservar su autonomía frente a las presiones de empleadores y clientes, garantizando a la vez que su conducta fuera acorde con lo establecido en el código ético profesional.

2. Potenciar el componente ético en los planes de formación de los profesionales. El razonamiento ético debería coexistir siempre con el económicocontable. En este sentido, si bien no sería recomendable fundir conocimientos científico-técnicos y éticos en la misma asignatura o materia, si lo sería establecer un equilibrio razonable en los planes de estudio entre disciplinas científicas y humanísticas, teniendo la ética, entre estas últimas, un peso especifico suficiente. 
3. Cambiar progresivamente la mentalidad social en la que aún abundan visiones sesgadas acerca del carácter técnico (amoral) y de segundo plano de la actividad contable, actividad a la que la opinión vulgar ve solo ocupada en asuntos de detalle, minúsculos y de escasa importancia. En este empeño sería importante incrementar la actividad investigadora y de divulgación de los propios profesionales, convirtiéndolos en abogados de su propia profesión tanto frente a sus propios clientes, como hacia el público en general e incluso hacia aquella parte de la propia profesión contable que permanece aún ciega a la auténtica dimensión y responsabilidad de su tarea.

\section{BIBLIOGRAFÍA.}

AMAT, O. y OLIVERAS, E. (2004), "Propuestas para combatir la contabilidad creativa", Universia Business Review, Primer trimestre 2004, pp.10-17.

BENSTON, G. (2003), "The Quality of Corporate Financial Statements and Their Auditors Before and After Enron", Policy Analysis, num 497 , November 6, 2003, pp. 1-29.

BYRNE, N. y otros (2002), "Accounting in Crisis", BusinessWeek, January 28, 2002, pp.44-48.

CERNUSKA, L. (2011), "Ethics in Accounting: The Consequences of Enron Scandal", Lucrari Stiintifice Seria I, Vol. 13, Issue 3, p. 35-42.

CLAYPOOL, G. y otros (1990), "Reactions to Ethical Dilemmas: A Study Pertaining to Certified Public Accountants", Journal of Business Ethics, Sept. 1990, Vol.9, Issue 9, pp. 699-706.

COASE, R. (2002), "Why Economics Will Change", ISNIE Newsletter, Summer 2002, Volume 4, num. 1, pp. 1-7.

DOGAN, M y PAHRE, R. (1989), "Fragmentation and Recombination of Social Sciences", Studies in Comparative International Development, Summer 89, Vol. 24, Issue 2, pp. 56-73.

DUSKA, R y otros (2011), Accounting Ethics, Wiley-Blackwell, Oxford.

FRIEDMAN, M. (1953), "Methodology of Positive Economics" en Friedman M. Essays in Positive Economics, University Chicago Press, Chicago, pp.3-43.

GOWTHORPE, A y BLAKE, J. (eds) (1998), Ethical Issues in Accounting, Routledge, London.

I.E.S.B.A. (2013), Handbook of the Code of Ethics for Professional Accountants. International Federation of Accountants, New York.

KLING, A. (2010), "The Root of the Financial Crisis", Policy Review, December 2009-Jan.2010, pp. 21-33.

KOTHARI, S. y LESTER, R. (2012), "The Role of Accounting in the Financial Crisis: Lessons for the Future”, Accounting Horizons, vol.26, num. 2, pp. 335-351. 
LOWENSTEIN, R (2008). “Triple A Failure” New York Times. April, 27, 2008. Disponible en http://www.nytimes.com/2008/04/27/magazine/27Credit-t. html?pagewanted=all\&_r=0

McPHAIL, K. (2009), Accounting Eु Business Ethics, Routledge, USA-Canadá. MITCHEL, W. (1923), "Business Cycles", en National Bureau of Economic Research, Business Cycles and Unemployment, Committee of de President's Conference of Unemployment, pp. 7-20.

NIELSEN, R. (2010), "High Leverage Finance Capitalism, the Economic Crisis, Structurally Related Ethics Issues, and Potential Reforms”, Business Ethics Quarterly, Apr.2010, vol. 20, Issue 2, pp. 299-330.

O'REILLY, E. (2011),"Are You Capitalising on Your Data Assets", Accountancy Ireland, Apr.2011, vol. 43, Issue 2, pp. 26-27.

PELÁEZ, E. (2011), "Crisis financiera y crisis económica", Cuadernos de Formación, Colaboración 8/11, volumen 12/2011, pp.111-129.

PIAGET, J. (1970), "The Place of the Sciences of Man in the System of Sciences" en Main Trends of Research and Human Sciences. Part One: Social Sciences, Mouton/UNESCO, Paris/The Hague.

RAMPELL, C. (2010), “The 'Great Recession' Earns Its Title”, New York Times, July 30.

SOIM, H. (2012), "The Causes of Economic Crisis. A Behavioral Foundation", Annals of the University of Oradea. Economic Science Series, vol.21, Issue 1, pp 421-426.

VICKERS, D. (1997), Economics and Ethics, Greenwood, Westport, Connecticut. 